\title{
High Frequency-Low Loss SAW Resonators Built on NanoCrystalline Diamond-Based Substrate
}

\author{
L. Braun, P. Boillot, S. Renaud, E. Girardet \\ AR Electronique SAS \\ Besançon, France \\ G. Martin, R. Salut, W. Daniau \\ FEMTO-ST, UMR 6174 CNRS-UFC-ENSMM-UTBM \\ Besançon, France \\ C. Gesset, P. Bergonzo, S. Saada \\ CEA LIST, Diamond Sensors Laboratory, CEA-Tech \\ Gif-sur-Yvette, France
}

\author{
E. Courjon, T. Laroche, S. Ballandras \\ Frec $|n|$ sys SAS \\ Besançon, France \\ S. Desgrez, L. Raynaud \\ THALES Alenia Space \\ Toulouse, France
}

\author{
K. Ait Aissa, O. Legrani, F. Sarry, B. Vincent, \\ O. El Mazria \\ IJL, UMR 7198 CNRS-Université de Lorraine \\ Vandœuvre-lès-Nancy, France
}

\author{
S. Laurent, J.-C. Nallatamby \\ XLim-Brive, UMR 6172 CNRS-Université de Limoges \\ Brive, France
}

\begin{abstract}
The interest of surface acoustic wave devices (SAW) operating in radio-frequency range is their very high compactness, low losses and high quality factor. Thus, they are very interesting components for the stabilization of ultra-low noise on-board oscillators operating in direct bands. The need for working frequency beyond $3 \mathrm{GHz}$ has lead SAW manufacturers to develop multilayer diamond-based waveguides providing higher phase velocity than conventional single-crystal materials, typically ranging from 7 to $12 \mathrm{~km} . \mathrm{s}^{-1}$ for elliptically polarized waves. The very problem in this approach is the excitation and detection of acoustic waves requiring a high quality piezoelectric layer of less than one micrometer thick with physical properties as close as possible to the tabulated ones. An ultimate control of the film thickness and roughness is required to control dispersion and diffusion losses.
\end{abstract}

In this work, we investigated a structure based on NanoCrystalline Diamond (NCD). The waves are launched and detected using a Zinc Oxide film deposited atop the diamond layer, yielding notable dispersive properties of the device. In this way, technological developments have been achieved (NCD growth, piezoelectric layer deposition, e-beam and optical lithography) to build SAW devices taking advantage of NCD films to try and benefit from their suspected low acoustic damping. Results show the possibility of developing devices operating between 2 and $3 \mathrm{GHz}$ at minimum, having losses lower than $10 \mathrm{~dB}$. At last, devices whose dimensions are compatible with conventional lithography processes, show resonances at more than $4 \mathrm{GHz}$ with less than $8 \mathrm{~dB}$ of insertion loss, what is, at the knowledge of the authors, the best experimental result for such devices. To compare with previous results, a device operating near $3 \mathrm{GHz}$ has been used to stabilize an oscillator. Short term stability has been measured at $10^{-8} \mathrm{~s}^{-1}$ and a phase noise floor was observed at $-170 \mathrm{~dB}$ at $3 \mathrm{MHz}$ from the carrier. Although not yet meeting the expected requirements, these results show the impact of loss reduction and general device improvement and allow for preparing future work near $5 \mathrm{GHz}$.

Keywords-Surface Acoustic Wave; Thin Layers; NanoCrystal Diamond; ZnO; Radio-Frequency; E-beam Lithography; Low Loss

\section{INTRODUCTION}

A lot of work already has been dedicated to evaluate Diamond-based substrates for the development of high frequency Surface Acoustic Wave (SAW) devices for various Radio-Frequency (RF) applications [1][2][3]. The main interest of such material consists in its very high acoustic phase velocities, typically ranging from 9 to $12 \mathrm{~km} . \mathrm{s}^{-1}$ when used as SAW wave guide. The absence of any piezoelectric properties of this material however requires the use of a piezoelectric film to excite and detect the waves. As a consequence, the quality of the final device exploiting such material directly depends on the quality of the material stack. In this work, the use of Nano-Crystalline Diamond (NCD) is investigated for the fabrication of surface acoustic wave guides and more particularly resonators based on such materials, expecting to improve the device operation by reducing intrinsic loss mechanisms compared with standard Diamond-Like-Carbon (DLC)-based devices. A simple material combination has been considered in that purpose, i.e. Zinc Oxyde $(\mathrm{ZnO})$ deposited using sputtering technique on 5 to $20 \mu \mathrm{m}$ thick NCD layers grown using Microwave Plasma assisted Chemical Vapor Deposition (MPCVD). The selfsupported NCD substrate is built along the process principle proposed in [6]. In this work, the impact of the surface quality of the substrate is emphasized for achieving state-of-the-art resonators (Qf 4.10 ${ }^{12}$ at $4 \mathrm{GHz}$ ). Numerous resonators have 
been fabricated on such substrates, allowing for characterizing resonances between 2 and $4 \mathrm{GHz}$. The devices were fully characterized to identify the effective capabilities of such a structure to enter the composition of radio-frequency (RF) filters operating in the $\mathrm{S}$ and $\mathrm{C}$ bands, at frequency upper than those usually considered for standard SAW industry production capabilities.

In the first section of the paper we briefly recall the chosen waveguide configuration, showing the dispersion analysis used to design the resonator. Information about the NCD is briefly reported, and the piezoelectric film and the Electron Beam Lithography (EBL) processes are recalled. Characterization results of double-port resonators also are presented and the resonators characteristics are reported. Packaged resonators were used to stabilize an oscillating loop. Its phase noise and short-term stability are reported.

\section{BASIC PRINCIPLE}

Although many configurations can be achieved, the simplest one was preferred as an effective implementation of the designed devices. In that purpose, tricky material combinations requiring numerous etching and/or deposition steps have been avoided that may prevent the achievement of test vehicles. Figure 1 shows the selected structure and defines axis orientations and layer dimensions.

The piezoelectric layer considered to excite and detect the guided waves is $\mathrm{C}$-axis-oriented $\mathrm{ZnO}$. The principal wave parameters then have been computed from the effective permittivity of the material stack top surface. Only non-leaky waves are considered in the present study for reducing as much as possible insertion losses of final devices. Dispersion curves for the selected material configuration are reported in Figure 2.

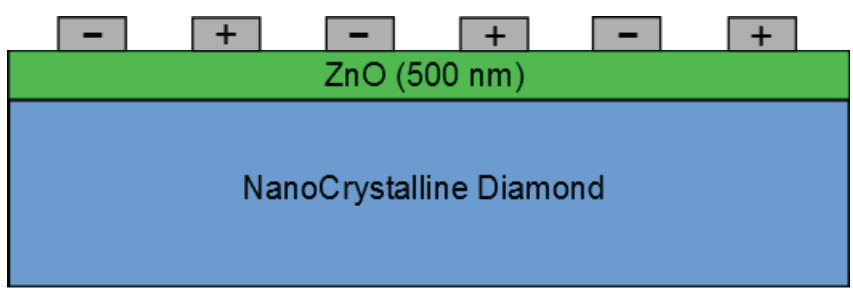

Figure 1: Scheme of the waveguide/transducer basic structure

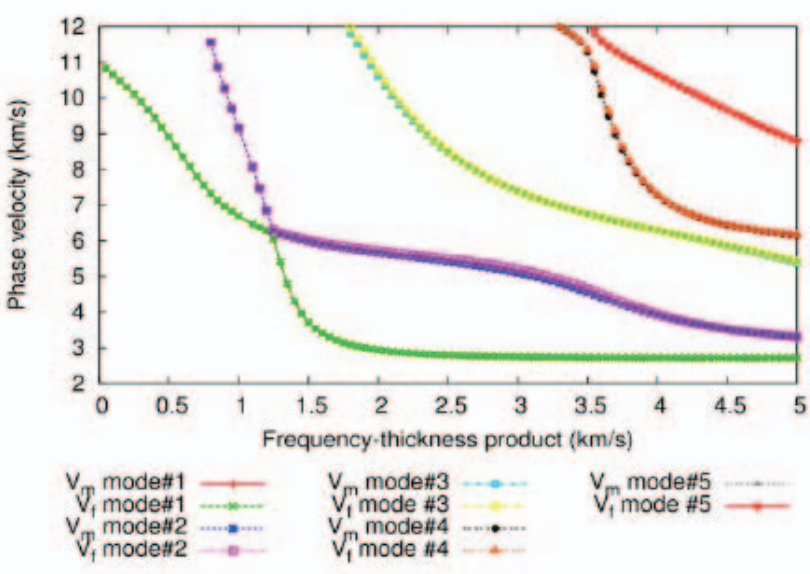

Figure 2: Dispersion curves of the 5 first modes on a $\mathrm{ZnO} / \mathrm{NCD} / \mathrm{Si}$ material stack

\section{WAVEGUIDE MANUFACTURING}

NCD layers are grown thanks to Bias Enhanced Nucleation-Microwave Plasma assisted Chemical Vapor Deposition (BEN-MPCVD) [4]. In this process two types of gases are exploited, Hydrogen $\left(\mathrm{H}_{2}\right)$ and Methane $\left(\mathrm{CH}_{4}\right)$. The standard growth process corresponds to a plasma powers between 600 to $2000 \mathrm{~W}$, a pressure between 20 to $100 \mathrm{mbar}$ and a substrate temperature between 500 to $1000^{\circ} \mathrm{C}$. The XPS spectrum of the achieved layers shows a high contribution (77\%) of the carbon $\mathrm{sp} 3$ (characteristic of the diamond), and only $23 \%$ of a less organized phase [5]. Although rather small roughness were obtained ( 1 to $4.5 \mathrm{~nm}$ RMS), the use of nucleation surfaces was then preferred to simultaneously meet roughness compatible with SAW requirements and optimal EBL conditions. Nine resonators are built on a given sample, yielding statistical assessment of the device operation. After lift-off, the devices are characterized thanks to a high resolution SEM, and 2 pictures are presented in Figure 3.
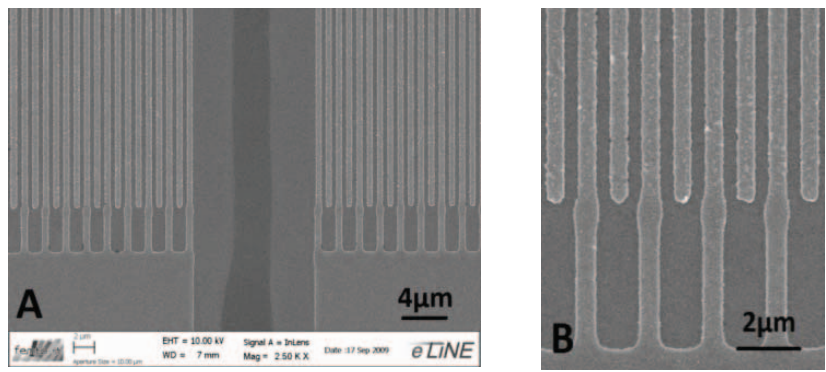

Figure 3: SEM pictures of a double port resonator - A: view near the cavity, $\mathrm{B}$ : close view of IDT fingers

\section{EXPERIMENTAL CHARACTERIZATION}

First results were obtained with double-port resonators with $2 \mu \mathrm{m}$ wavelength and are presented on figure Figure 4. Their response, measured in transmission, show resonances at 2.2 and $2.85 \mathrm{GHz}$ for modes 1 and 2 respectively. This last exhibits insertion loss of about $10 \mathrm{~dB}$, what is quite remarkable. However Q-factor is lower than expected with 400 . We also find a contribution at $5.5 \mathrm{GHz}$, but as for mode 1 , insertion loss exceeds $20 \mathrm{~dB}$. 


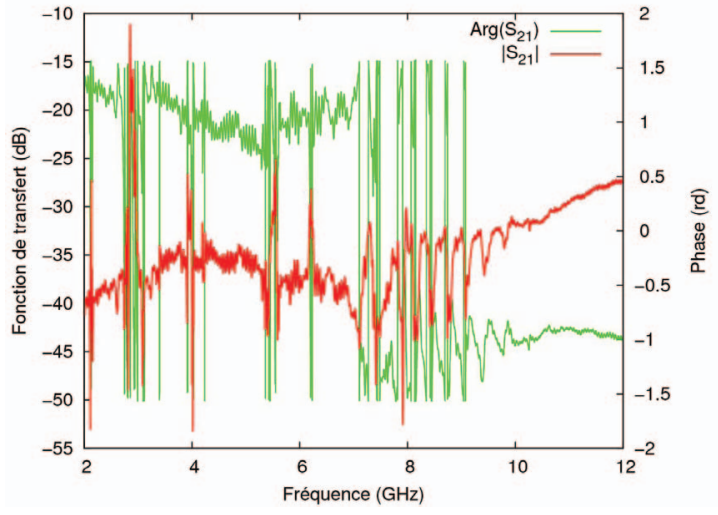

(a)

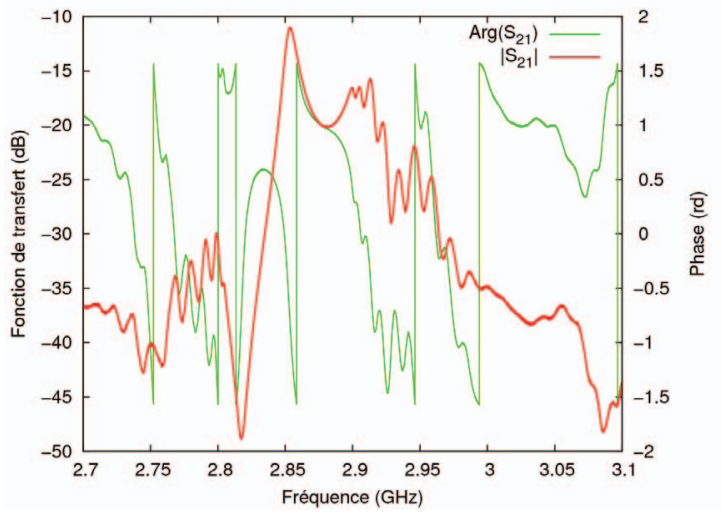

(b)

Figure 4: Transfer function of double port resonators with $2 \mu \mathrm{m}$ wavelength (a) large view, (b) view closed to resonance of mode 2

A second sample of resonators was characterized. There wavelength reach about $1 \mu \mathrm{m}$, what is compatible with resolution of optical lithography equipment using i-line sources. A typical response is shown on Figure 5. Mode 2 is well defined at $4.2 \mathrm{GHz}$ with less than $8 \mathrm{~dB}$ insertion loss, what is a state-of-the art result. But as for $2 \mu \mathrm{m}$ wavelength devices, Q-factor is lower than the prevision, with 250. Contribution of mode 3 is also visible at $7 \mathrm{GHz}$, but its low rejection level $(8 \mathrm{~dB})$ and high insertion loss $(25 \mathrm{~dB})$ make it difficult to use in a feed-back oscillator fabrication.

A sample of resonators has been packaged. Their transfer functions are shown on Figure 6 for modes 1 and 2. Oscillators locked on these two modes have been realized (Figure 7). Phase noise measurements (Figure 8) show an important $\mathrm{f}^{-3}$ noise contribution and levels of -70 and $-65 \mathrm{dBc} / \mathrm{Hz}$ at $1 \mathrm{kHz}$ away of the carrier, for modes 1 and 2 respectively. Noise floor levels have been measured at -165 and $-170 \mathrm{dBc} / \mathrm{Hz}$ respectively.

Their Allan variances have also been measured from 0.1 to 100 s, for both modes and are summed up in Table 1.

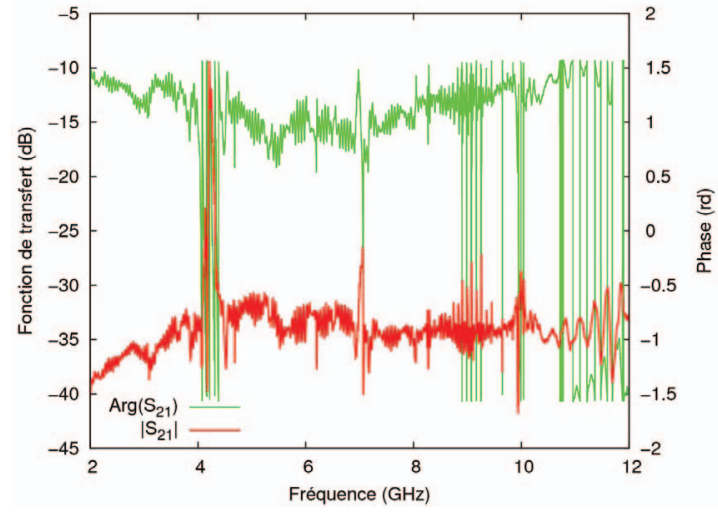

(a)

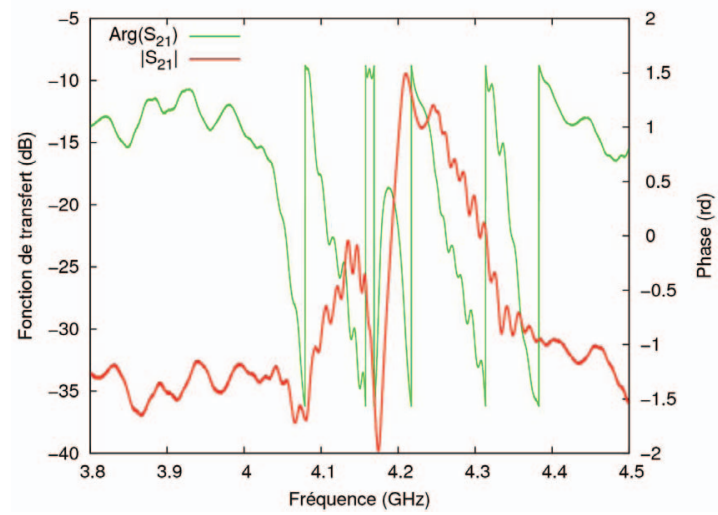

(b)

Figure 5: Transfer function of double port resonators with $1 \mu \mathrm{m}$ wavelength (a) large view, (b) view closed to resonance of mode 2

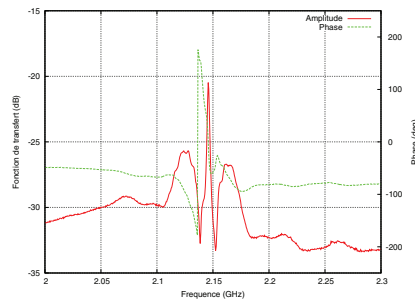

(a)

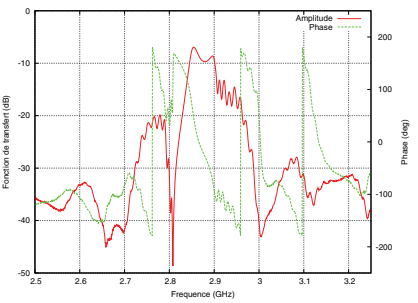

(b)
Figure 6: Transfer functions of $2 \mu \mathrm{m}$ wavelength resonators after packaging (a): mode $1,(\mathrm{~b})$ : mode 2

\section{CONCLUSION}

In this work, high frequency resonators have been developed for frequency source applications. These resonators have been manufactured using Electron Beam Lithography on $\mathrm{ZnO} /$ Diamond substrate, exploiting the nucleation side of the NCD exhibiting small arithmetic averaged roughness $(1 \mathrm{~nm}$ RMS at best) compatible with a reliable and reproducible lithography step. 


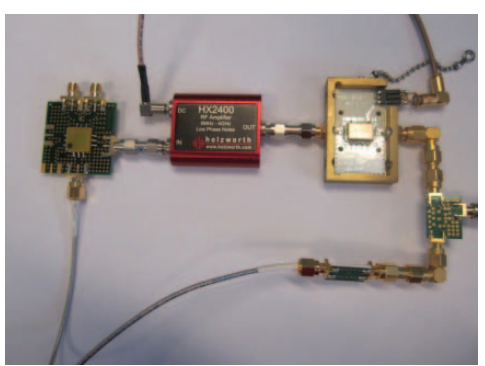

Figure 7: Picture of the oscillator circuit

Nano-Crystalline Diamond films and substrates have also been used to minimize the acoustic losses expected in standard poly-crystal diamond-like carbon films due to large grain overlays [4], expected to favor acoustic diffusion along the surface propagation. Double port resonators, have been built in the 2-5 GHz frequency range with insertion loss lower than $10 \mathrm{~dB}$. Oscillators have been built using modes at 2.2 and $2.85 \mathrm{GHz}$, showing phase noise floor at $-170 \mathrm{dBc} / \mathrm{Hz}$. Whatever, more efforts are required, mainly to improve Qfactor and to optimize the oscillation loop. The perspective of making devices on 4 inch wafers with conventional photolithography equipment will be also considered.

TABLE I. ALLAN VARIANCE MEASURE FOR OSCILLATORS AT 2.2 AND $2.85 \mathrm{GHz}$

\begin{tabular}{|c|c|c|c|c|}
\cline { 2 - 5 } \multicolumn{1}{c|}{} & $0.1 \mathrm{~s}$ & $1 \mathrm{~s}$ & $10 \mathrm{~s}$ & $100 \mathrm{~s}$ \\
\hline $2.2 \mathrm{GHz}$ & $3.6 .10^{-8}$ & $2.9 .10^{-8}$ & $6.2 .10^{-8}$ & $5.8 .10^{-7}$ \\
\hline $2.85 \mathrm{GHz}$ & $4.3 .10^{-8}$ & $4.9 .10^{-8}$ & $1.2 .10^{-7}$ & $1.0 .10^{-6}$ \\
\hline
\end{tabular}

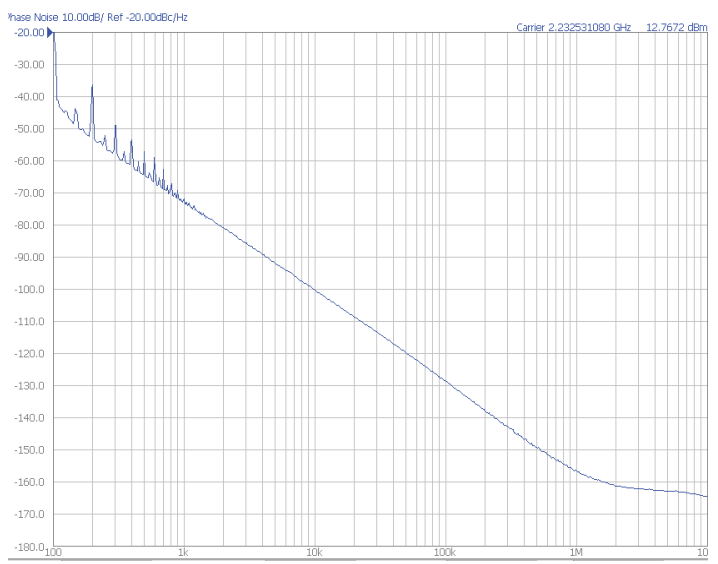

(a)

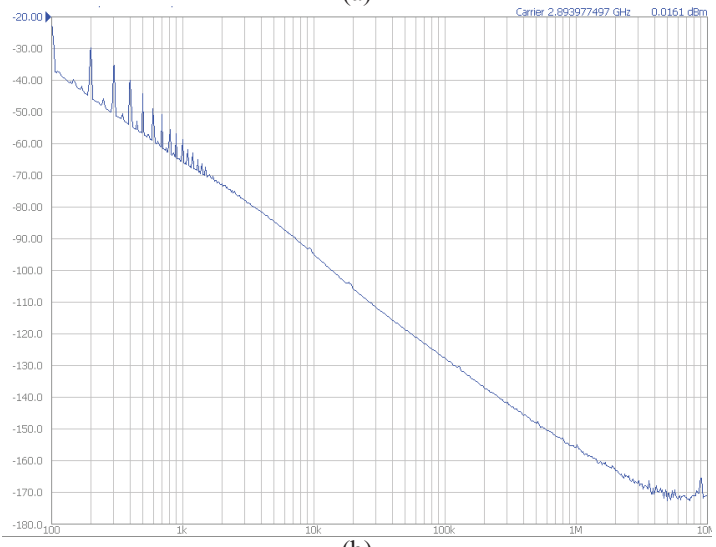

(b)

Figure 8: Phase noise characteristics of feed-back oscillators locked on mode 1 (a) at $2.2 \mathrm{GHz}$ and mode 2 at $2.85 \mathrm{GHz}$ (b) of a $2 \mu \mathrm{m}$ wavelength resonator

\section{ACKNOWLEDGMENT}

This work is supported by the Direction Générale de l'Armement (DGA) under grant\#6369740680238016.

\section{REFERENCES}

[1] A. Hachigo et al," Proc. of the IEEE ultrasonics symposium, pp. 325328,1999

[2] M. Pereira da Cunha et al, Proc. of the IEEE Ultrasonics Symp., vol. 1, pp. 283-286, 2000.

[3] H. Nakahata et al., Proc. of the IEEE ultrasonics symposium, pp. 319$322,1998$.

[4] J.C. Arnault et al, Chemical vapor deposition, vol. 14, no. 7-8, pp. 187195, 2008.

[5] S.Saada et al, phys. stat. sol. (a) 205, No. 9, 2121-2125, 2008.

[6] V. Mortet et al, Appl. Phys. Lett. 81, 1720 (2002); http://dx.doi.org/10.1063/1.1503875 (3 pages) 